\title{
Hu-Lu-Ba-Wan Attenuates Diabetic Nephropathy in Type 2 Diabetic Rats through PKC- $\alpha$ /NADPH Oxidase Signaling Pathway
}

\author{
Lishan Zhou, ${ }^{1,2}$ Hui Dong, ${ }^{1}$ Yi Huang, ${ }^{3}$ Lijun Xu, ${ }^{1}$ Xin Zou, ${ }^{1}$ Kaifu Wang, \\ Guang Chen, ${ }^{4}$ and Fuer Lu ${ }^{1}$ \\ ${ }^{1}$ Institute of Integrated Traditional Chinese and Western Medicine, Tongji Hospital, Tongji Medical College, \\ Huazhong University of Science and Technology, Wuhan, Hubei 430030, China \\ ${ }^{2}$ Department of Integrated Traditional Chinese and Western Medicine, Wuhan Children's Hospital, \\ Wuhan, Hubei 430016, China \\ ${ }^{3}$ Department of Nephrology, Tongji Hospital, Tongji Medical College, Huazhong University of Science and Technology, \\ Wuhan, Hubei 430030, China \\ ${ }^{4}$ Department of Integrated Traditional Chinese and Western Medicine, Tongji Hospital, Tongji Medical College, \\ Huazhong University of Science and Technology, Wuhan, Hubei 430030, China
}

Correspondence should be addressed to

Guang Chen; guangchen8086@126.com and Fuer Lu; felu@tjh.tjmu.edu.cn

Received 18 January 2013; Revised 18 May 2013; Accepted 19 May 2013

Academic Editor: Musa T. Yakubu

Copyright @ 2013 Lishan Zhou et al. This is an open access article distributed under the Creative Commons Attribution License, which permits unrestricted use, distribution, and reproduction in any medium, provided the original work is properly cited.

\begin{abstract}
$\mathrm{Hu}-\mathrm{Lu}-\mathrm{Ba}-W a n(\mathrm{HLBW})$ is a Chinese herbal prescription used to treat kidney deficiency. The aim of this study was to explore the effect and mechanism of HLBW on diabetic nephropathy (DN) in type 2 diabetic rats. The rat model of DN was established by being fed a high-fat diet and intravenous injection of streptozotocin. Then, HLBW decoction was administered for 16 weeks. Blood glucose level, lipid profile, renal function, 24-hour total urinary protein, and albumin content were examined. Renal morphology and superoxide anion levels were evaluated. The activity of nicotinamide-adenine dinucleotide phosphate (NADPH) and protein kinase C-alpha (PKC- $\alpha$ ) related genes expression in renal tissue were also determined. Our data demonstrated that HLBW significantly improved hyperglycemia, hyperlipidemia, and proteinuria in diabetic rats compared with those of control group. HLBW also alleviated glomerular expansion and fibrosis, extracellular matrix accumulation and effacement of the foot processes. Additionally, HLBW reduced superoxide anion level, NADPH oxidase activity, the protein and mRNA expressions of p4 $7^{\text {phox }}$, and the protein expression of phosphorylated PKC- $\alpha$ in renal tissue. These results suggest that HLBW is effective in the treatment of DN in rats. The underlying mechanism may be related to the attenuation of renal oxidative stress via PKC- $\alpha / \mathrm{NADPH}$ oxidase signaling pathway.
\end{abstract}

\section{Introduction}

The epidemic of diabetes mellitus (DM) is rapidly becoming a severe public health problem, especially in China. It is reported that the prevalence of diabetes has reached 9.7\%, accounting for 92.4 million adults with diabetes [1]. The morbidity and mortality associated with this disease derives primarily from its microvascular and macrovascular complications caused by persistent hyperglycemia [2]. Diabetic nephropathy (DN), with hidden symptoms in early stage and lack of effective therapy, is the most common microvascular complication of diabetes [3]. Accumulating evidence has demonstrated that hyperglycemia and the resulting oxidative stress play important roles in the development of DN [4-6]. Therefore, therapies designed to improve hyperglycemia and reduce oxidase stress may be promising in the treatment of $\mathrm{DN}$.

Plant-based medicines are widely used to treat diabetes and its complications in the local clinics of China. In Chinese medicine, DN is referred to kidney deficiency disease. Therefore, prescriptions reinforcing kidney may have the potential to treat DN. Among effective prescriptions, Hu-Lu-Ba-Wan 
TABLE 1: Real-time PCR primer sequences.

\begin{tabular}{|c|c|c|}
\hline Gene & Forward $\left(5^{\prime} \rightarrow 3^{\prime}\right)$ & Reverse $\left(5^{\prime} \rightarrow 3^{\prime}\right)$ \\
\hline$\beta$-Actin & $5^{\prime}$-GGAGATTACTGCCCTGGCTCCTA-3' & $5^{\prime}$-GACTCATCGTACTCCTGCTTGCTG-3' \\
\hline $\mathrm{p} 47^{\text {phox }}$ & 5'-GGACACCTTCATTCGCCACA-3' & $5^{\prime}$-GTCCTGCCACTTAACCAGGAACA-3' \\
\hline PKC- $\alpha$ & $5^{\prime}$-TCCAGGATGACGACGTGGAG-3' & 5'-CGTTGACGTATTCCATGACGAAG-3' \\
\hline
\end{tabular}

TABLE 2: The effect of HLBW on OGTT in rats with DN.

\begin{tabular}{|c|c|c|c|}
\hline Group & $\mathrm{FBG}(\mathrm{mmol} / \mathrm{L})$ & PG-1h (mmol/L) & PG-2 h $(\mathrm{mmol} / \mathrm{L})$ \\
\hline Control & $5.84 \pm 0.55$ & $6.15 \pm 0.37$ & $6.05 \pm 0.60$ \\
\hline Diabetic & $15.81 \pm 1.99^{\triangle \Delta}$ & $27.10 \pm 4.52^{\triangle \Delta}$ & $23.29 \pm 3.77^{\triangle \Delta}$ \\
\hline TFG & $5.76 \pm 0.44^{\mathbf{\Lambda}}$ & $10.11 \pm 2.97^{\mathbf{\Lambda}}$ & $6.51 \pm 1.32^{\mathbf{\Lambda}}$ \\
\hline PC & $5.75 \pm 1.30^{\Lambda \Lambda}$ & $9.81 \pm 3.71^{\boldsymbol{\Lambda}}$ & $6.26 \pm 1.13^{\Delta \Lambda}$ \\
\hline HLBW & $5.73 \pm 1.01^{\Lambda \Lambda}$ & $8.08 \pm 0.91^{\mathbf{\Lambda}}$ & $6.01 \pm 0.91^{\mathbf{\Lambda}}$ \\
\hline Captopril & $8.50 \pm 1.58^{\mathbf{\Lambda}}$ & $14.90 \pm 5.78^{\Lambda \Lambda}$ & $9.84 \pm 1.64^{\boldsymbol{\Lambda}}$ \\
\hline
\end{tabular}

Values are mean $\pm \mathrm{SD}(n=8) .{ }^{\triangle} P<0.01$ versus the control group, $\triangle^{\Delta} P<0.01$ versus the untreated diabetic group. FBG: fasting blood glucose; PG-1 h: postprandial blood glucose at 1 hour after glucose loading; PG-2 h: postprandial blood glucose at 2 hours after glucose loading.

(HLBW), which consisted of Trigonella foenum-graecum L. (TFG) and Psoralea corylifolia L. (PC), attracts much attention for their novel kidney reinforcing efficacy. Many researches have shown the beneficial effect of TFG and PC on improving hyperglycemia and alleviating inflammation [7-13]. In a recent, their antioxidative activity and renal protective effect have also been documented [14-19]. However, these individual studies just included incomplete description in this aspect. The effect of HLBW, which is the combination of TFG and PC, on DN is rarely reported. Therefore, the aim of this study was to explore the effect of HLBW on DN in type 2 diabetic rats and investigate its possible oxidative stress against mechanism.

\section{Materials and Methods}

2.1. Animals. Male Wistar rats weighting $180 \sim 200 \mathrm{~g}$, obtained from Hubei province Center for Disease Control and Prevention, were used in this study. The rats were maintained at ambient temperature $\left(22^{\circ} \mathrm{C} \pm 1^{\circ} \mathrm{C}\right)$ with a $12: 12 \mathrm{~h}$ light-dark cycle and free access to water and food. The research was conducted in accordance with the internationally accepted principles for laboratory animal use and care as found in the Chinese guidelines.

2.2. Preparation of $H L B W$. TFG and PC were purchased from Traditional Chinese Medicine Company in Hubei Province (Wuhan, China) and authenticated by the Department of Pharmacognscy, Hubei University of Chinese Medicine (Wuhan, China). The rat doses of TFG and PC were obtained by the conversion of human doses (Chinese Pharmacopeia, 2010) to rat equivalent doses based on body surface areas. The weight ratio of TFG to PC is $1: 1$. HLBW preparation process was as follows. The dry seeds were crushed into powder and soaked overnight before boiled. After cooling down, the decoction was deposited in 95\% ethanol (1:1 v/v) overnight at room temperature. The precipitate from the decoction was separated by a filter. Then, the alcoholic filtrate was distilled to remove the ethanol and was concentrated by Rotavapor (BUCHI, Flawil, Switzerland). The final liquid extracted from $1 \mathrm{~g}$ HLBW (which contains $0.5 \mathrm{~g}$ TFG and $0.5 \mathrm{~g}$ PC), $1 \mathrm{~g}$ TFG, and $1 \mathrm{~g} \mathrm{PC}$ was $1.32 \mathrm{~mL}, 0.63 \mathrm{~mL}$, and $0.66 \mathrm{~mL}$, respectively.

2.3. Animal Modeling, Grouping, and Treatment. After adaptive feeding with standard rat diet (containing 35\% flour, 20\% soy meal, $20 \%$ corn meal, $15.5 \%$ bran, $0.5 \%$ bean oil, $5 \%$ fish meal, $2.5 \%$ bone meal, $1 \%$ dusty yeast, and $0.5 \%$ salt) for one week, eight rats were selected randomly as the normal control group (control). The rats in the control group continued their standard diet, while the remaining rats were fed with a highfat diet (containing 67.5\% standard laboratory rat chow, 15\% lard, $15 \%$ sugar, $2 \%$ cholesterol, and $0.5 \%$ bile salts) for 4 weeks. Then, they were injected with streptozotocin (STZ, Sigma Chemical Co. MO, USA, $30 \mathrm{mg} / \mathrm{kg}$ ) into the tail vein after an overnight fast. One week later, oral glucose tolerance test (OGTT) was performed. The 95\% range of confidence was calculated according to the plasma glucose levels of normal rats. Rats with impaired glucose tolerance (IGT) (Plasma glucose levels of rats at two time points were higher than the upper limit at two time points, or $20 \%$ higher than the upper limit at one time point.) were selected. Then the IGT rats were randomized into the untreated diabetic control group (Diabetic), TFG treated group (TFG), PC treated group (PC), HLBW treated group (HLBW), and Captopril (SinoAmerican Shanghai Squibb Pharmaceuticals Ltd., Shanghai, China) treated group (Captopril). Rats in the previous mentioned treatment groups were administered with corresponding therapy, TFG $(9 \mathrm{~g} / \mathrm{kg} / \mathrm{d}), \mathrm{PC}(9 \mathrm{~g} / \mathrm{kg} / \mathrm{d})$, HLBW $(18 \mathrm{~g} / \mathrm{kg} / \mathrm{d})$, or Captopril $(10 \mathrm{mg} / \mathrm{kg} / \mathrm{d})$, intragastrically for 16 weeks. Oral gavage was performed once a day between 8:00 and 10:00 a.m. Rats in untreated diabetic and normal control group were administered with the same volume of distilled water. Doses were adjusted to the body weight recorded once a week.

2.4. Sampling. All the animals were sacrificed at the end of the 16th week. Urine samples of 24 hours were collected by metabolic cages the day before sacrifice. Blood samples were obtained by aorta abdominalis puncture at the time of sacrifice. After centrifuging at $3000 \mathrm{r} / \mathrm{min}$ for $30 \mathrm{~min}$ at $4^{\circ} \mathrm{C}$, the specimen serum was collected and stored at $-20^{\circ} \mathrm{C}$ until analysis. Meanwhile, kidneys were quickly taken out by laparotomy and flushed with normal saline on ice. Then, the entire kidney mass was scaled. Part of the left renal samples was, respectively, fixed in $4 \%$ paraformaldehyde solution for paraffin embedding or fixed in $2.5 \%$ glutaraldehyde solution 
TABLE 3: The effect of HLBW on plasma lipid profiles in rats with DN.

\begin{tabular}{lcccc}
\hline Group & TG $(\mathrm{mmol} / \mathrm{L})$ & TC $(\mathrm{mmol} / \mathrm{L})$ & LDL-C $(\mathrm{mmol} / \mathrm{L})$ & HDL-C $(\mathrm{mmol} / \mathrm{L})$ \\
\hline Control & $1.04 \pm 0.16$ & $2.09 \pm 0.29$ & $0.51 \pm 0.15$ & $1.50 \pm 0.24$ \\
Diabetic & $3.48 \pm 1.14^{\triangle \Delta}$ & $7.91 \pm 1.53^{\triangle \triangle}$ & $4.46 \pm 1.57^{\triangle \Delta}$ & $1.13 \pm 0.18$ \\
TFG & $1.23 \pm 0.38^{\mathbf{\Delta}}$ & $4.73 \pm 0.74^{\mathbf{\Delta}}$ & $1.29 \pm 0.36^{\mathbf{\Delta}}$ & $1.40 \pm 0.36$ \\
PC & $1.49 \pm 0.19^{\mathbf{\Delta}}$ & $5.06 \pm 1.05^{\mathbf{\Delta}}$ & $1.71 \pm 0.51^{\mathbf{\Delta}}$ & $1.35 \pm 0.35$ \\
HLBW & $1.18 \pm 0.23^{\mathbf{\Delta}}$ & $4.33 \pm 0.59^{\mathbf{\Delta}}$ & $1.20 \pm 0.28^{\mathbf{\Delta}}$ & $1.46 \pm 0.29$ \\
Captopril & $1.96 \pm 0.80$ & $7.23 \pm 1.62$ & $2.84 \pm 0.76$ & $1.28 \pm 0.16$ \\
\hline
\end{tabular}

Values are mean $\pm \mathrm{SD}(N=8) .{ }^{\triangle} \mathrm{A} P<0.01$ versus the control group, ${ }^{\mathbf{\Delta}} P<0.05,{ }^{\mathbf{\Delta}} \mathrm{P}<0.01$ versus the untreated diabetic group. TG: triglyceride; TC: total cholesterol; LDL-C: low-density lipoprotein cholesterol; HDL-C: high-density lipoprotein cholesterol.

TABLE 4: The effect of HLBW on renal function and proteinuria of rats with DN.

\begin{tabular}{|c|c|c|c|c|c|}
\hline Group & Kidney/body weight (\%) & $\mathrm{BUN}(\mathrm{mmol} / \mathrm{L})$ & $\mathrm{SCr}(\mu \mathrm{mol} / \mathrm{L})$ & Urinary total protein $(\mu \mathrm{g} / 24 \mathrm{~h})$ & Urinary albumin $(\mu \mathrm{g} / 24 \mathrm{~h})$ \\
\hline Control & $0.42 \pm 0.04$ & $3.96 \pm 0.81$ & $146.86 \pm 12.39$ & $6.71 \pm 2.39$ & $0.08 \pm 0.03$ \\
\hline Diabetic & $0.64 \pm 0.12^{\triangle \Delta}$ & $11.46 \pm 1.50^{\triangle \Delta}$ & $229.61 \pm 30.10^{\Delta \Delta}$ & $50.79 \pm 7.41^{\triangle \Delta}$ & $3.54 \pm 1.19^{\triangle \Delta}$ \\
\hline TFG & $0.48 \pm 0.08^{\mathbf{\Lambda}}$ & $5.68 \pm 1.53^{\mathbf{\Lambda}}$ & $184.89 \pm 15.72^{\mathbf{\Lambda}}$ & $13.40 \pm 5.22^{\mathbf{\Lambda}}$ & $0.41 \pm 0.35^{\mathbf{\Lambda}}$ \\
\hline PC & $0.43 \pm 0.06^{\mathbf{\Lambda}}$ & $5.70 \pm 1.02^{\mathbf{\Lambda}}$ & $183.34 \pm 23.61^{\boldsymbol{\Lambda \Lambda}}$ & $17.30 \pm 8.30^{\mathbf{\Lambda}}$ & $0.54 \pm 0.37^{\mathbf{\Lambda}}$ \\
\hline HLBW & $0.42 \pm 0.04^{\mathbf{\Lambda}}$ & $4.88 \pm 0.93^{\mathbf{\Lambda}}$ & $165.16 \pm 16.56^{\mathbf{\Lambda}}$ & $10.74 \pm 6.62^{\mathbf{\Lambda}}$ & $0.31 \pm 0.21^{\mathbf{\Lambda}}$ \\
\hline Captopril & $0.45 \pm 0.06^{\mathbf{\Lambda}}$ & $6.69 \pm 1.78^{\Lambda \mathbf{4}}$ & $190.13 \pm 21.62^{\mathbf{\Lambda}}$ & $14.00 \pm 4.08^{\mathbf{\Lambda \Lambda}}$ & $0.40 \pm 0.28^{\mathbf{\Lambda}}$ \\
\hline
\end{tabular}

Values are mean $\pm \mathrm{SD}(n=8) .{ }^{\triangle} \mathrm{P}<0.01$ versus the control group, ${ }^{\mathbf{\Delta \Lambda}} P<0.01$ versus the untreated diabetic group. BUN: blood urea nitrogen; SCr: serum creatinine.

for transmission electron microscope (TEM), while the other part was prepared for frozen sections. The right renal samples were preserved at $-80^{\circ} \mathrm{C}$ until use.

2.5. OGTT and Biochemical Analysis. After the rats fasted for 12 hours, $50 \%$ glucose at a dose of $2 \mathrm{~g} / \mathrm{kg}$ was orally administered. Then, blood samples were collected from tail veins at 0 (prior to glucose loading), 60 and 120 minutes (after glucose loading). Blood glucose level was examined by glucoseoxidase method using a glucose monitor (LifeScan Inc., J\&J Company, Milpitas, CA, USA). Serum levels of total cholesterol (TC), triglycerides (TG), low-density lipoprotein cholesterol (LDL-C), high-density lipoprotein cholesterol (HDL$\mathrm{C})$, blood urea nitrogen (BUN), and serum creatinine (SCr) were determined using commercial reagents (Jiancheng Bioengineering Institute, Nanjing, China). Urinary total protein and albumin concentrations were measured by the immunoturbidimetric method using a biochemical analyzer (Roche, Basel, Switzerland).

2.6. Renal Histological Studies. The paraffin slides were stained with hematoxylin and eosin (HE) to evaluate the histology of glomerulus, periodic acid-schiff (PAS) to evaluate the thickening of glomerular basement membrane and hyperplasia of mesangium, and Masson's trichrome stain (Masson) to evaluate the fibrosis of glomerulus, which were all observed under optical microscope. The following criteria were used for scoring renal histology. A semiquantitative score (sclerosis index (SI)) was used to evaluated the degree of glomerulosclerosis [20]. Severity of sclerosis for each glomerulus was graded from 0 to $4+$ as follows: 0 represents no lesion, $1+$ represents sclerosis of $<25 \%$ of the glomerulus, while $2+, 3+$, and $4+$ represent sclerosis of $25 \%$ to $50 \%,>50 \%$ to $75 \%$, and $>75 \%$ of the glomerulus. The whole kidney average sclerosis index on one section was obtained by averaging scores from all glomeruli. Renal ultrastructure was also observed under a transmission electron microscope (FEI Tecnai $\mathrm{G}^{2} 12$, Holland).

2.7. Detection of Renal Superoxide Anion Levels. Dihydroethidium (DHE), an oxidant-sensitive probe, is widely used for detection of reactive oxygen species (ROS). Two products of DHE oxidation, ethidium and 2-hydroxyethidium, can bind to the nuclear DNA, thereby forming a strong red fluorescent complex [21]. Frozen sections of the kidney $(8 \mu \mathrm{m})$ were placed on glass slides and incubated with DHE $(10 \mathrm{mmol} / \mathrm{L}$, Beyotime Institute of Biotechnology, Shanghai, China) in a dark container at $37^{\circ} \mathrm{C}$ for $30 \mathrm{~min}$. After rinsing in PBS three times, the sections were viewed with an inverted microscope (Nikon, Tokyo, Japan) [22].

2.8. Measurement of Renal NADPH Activity. Renocortical tissues were homogenated and lyzed in mammal tissue protein extraction reagent. Then, the extracted protein was supplemented with protease inhibitor cocktail and phenylmethylsulfonylfluoride (PMSF) (Guge shengwu Technology Co., Wuhan, China). After centrifuged at $12000 \mathrm{r} / \mathrm{min}$ for 30 min at $4^{\circ} \mathrm{C}$, the supernatant was collected to quantify the protein concentration with BCA protein assay kit (Beyotime Institute of Biotechnology, Shanghai, China). Renal NADPH activity was measured using an NADPH Activity Quantification Kit (Genmed Scientifics Inc., Shanghai, China).

2.9. Western Blot Analysis. Renocortical extracts (100 $\mu \mathrm{g}$ protein) were mixed with sample buffer, boiled for $10 \mathrm{~min}$, and subjected to $10 \%$ SDS-PAGE gel $(100 \mathrm{v}, 2 \mathrm{~h})$. Separated 
Hematoxylin and eosin staining (HE)

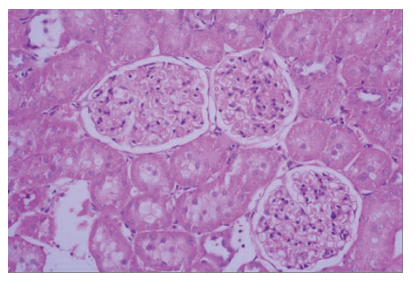

(a)

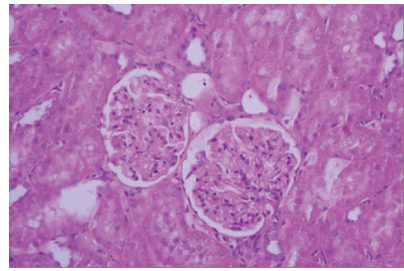

(d)

Periodic acid-schiff staining (PAS)

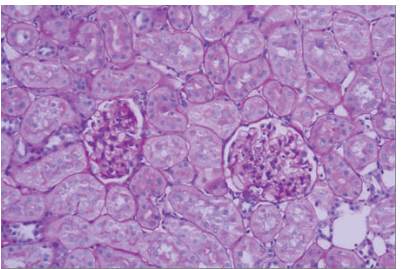

(a)

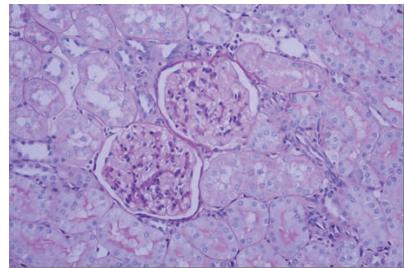

(d)

Masson's trichrome staining (Masson)

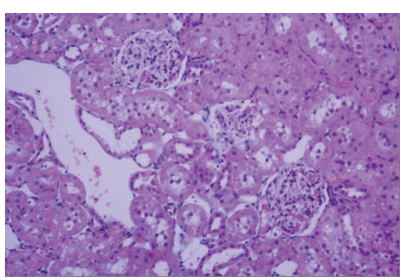

(a)

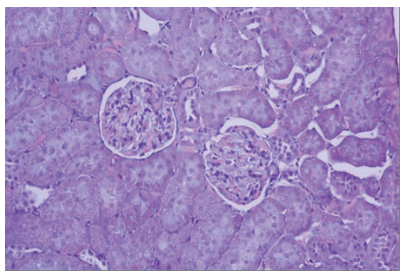

(d)

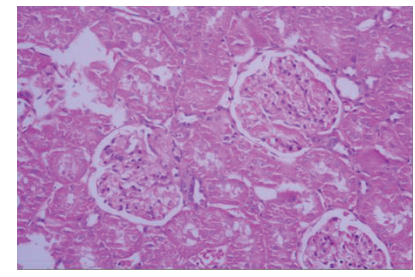

(b)

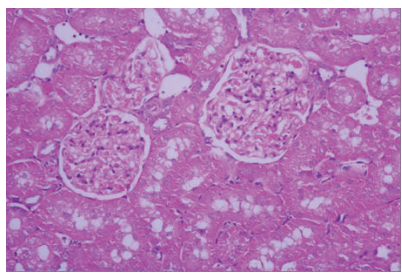

(e)

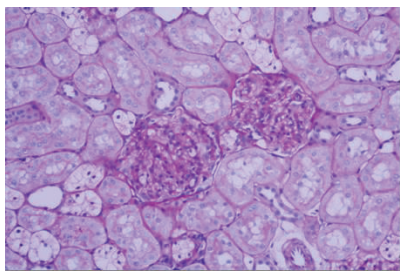

(b)

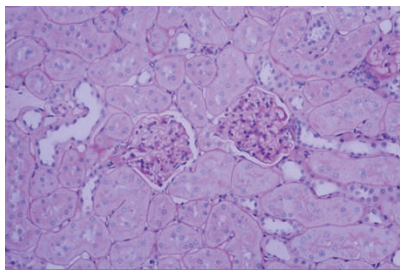

(e)

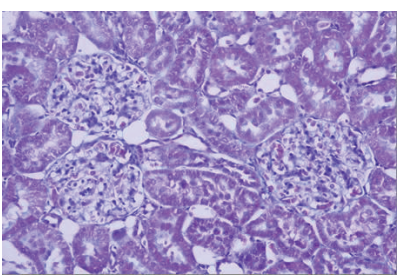

(b)

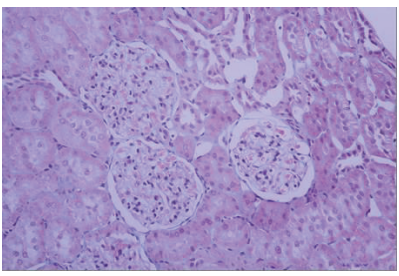

(e)

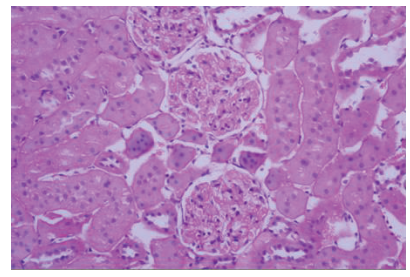

(c)

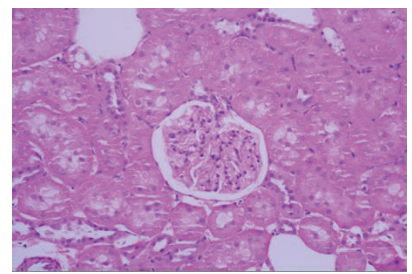

(f)

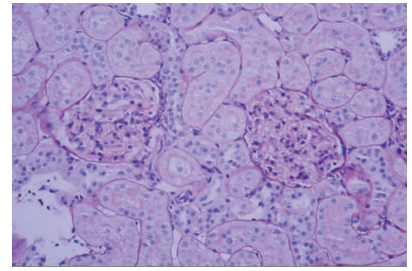

(c)

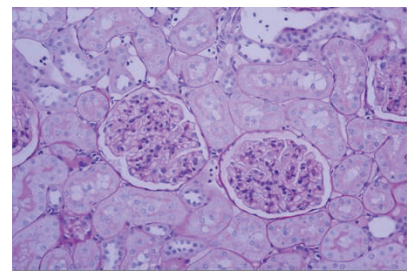

(f)

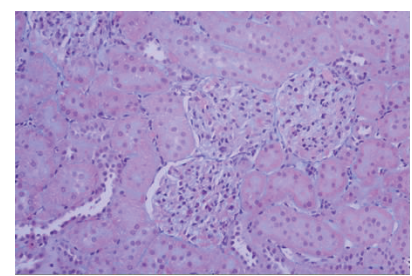

(c)

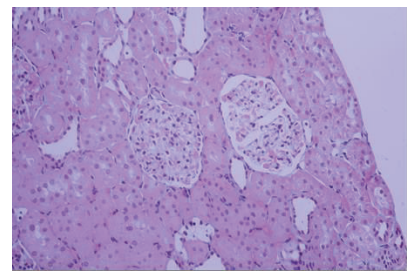

(f)

FIgURE 1: Continued. 


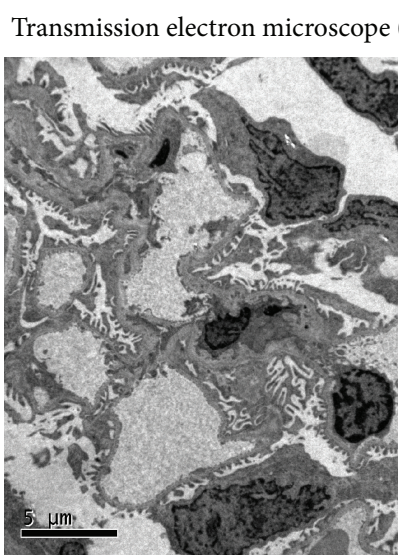

(a)

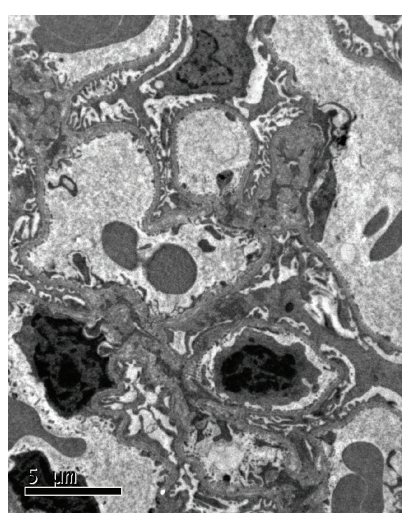

(d)

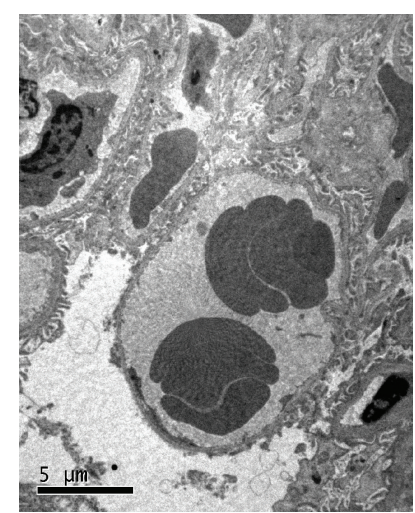

(b)

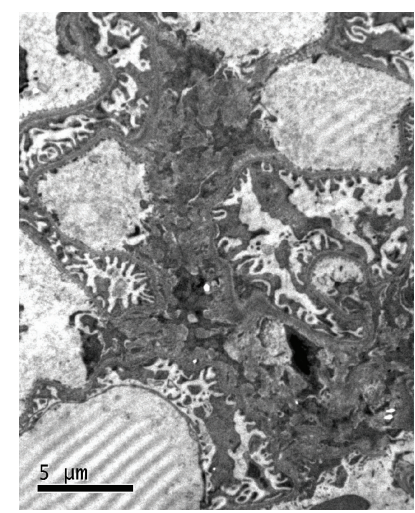

(e)

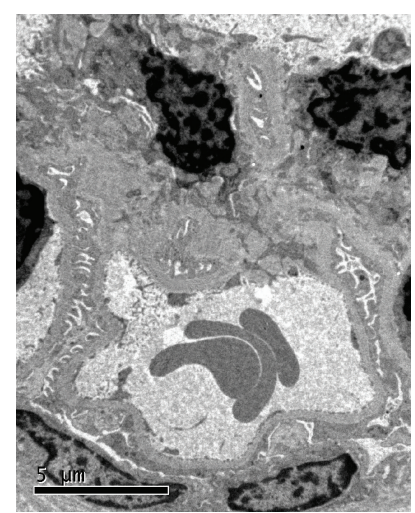

(c)

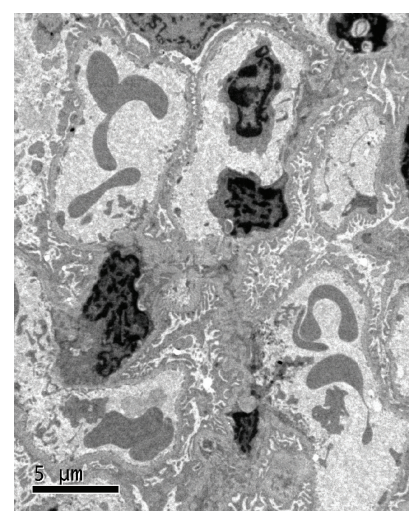

(f)

FIgURE 1: Morphological pictures of the glomerulus. (a) Control group; (b) Diabetic group; (c) TFG group; (d) PC group; (e) HLBW group; (f) Captopril group.

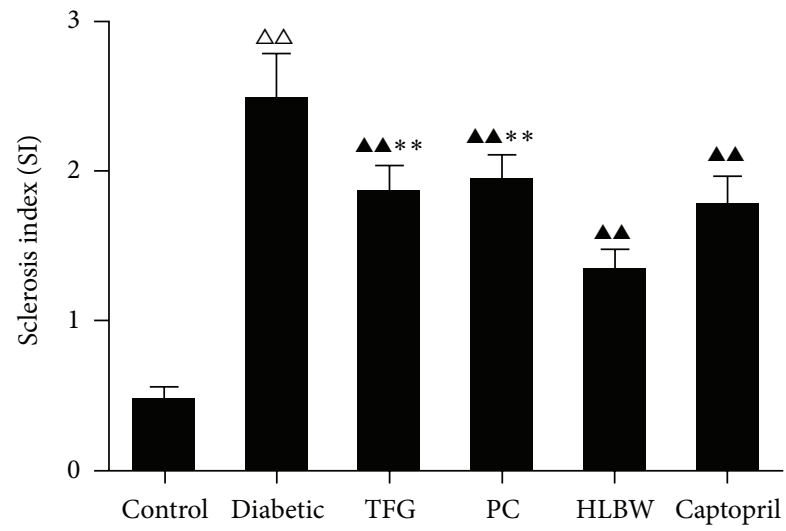

FIGURE 2: The effect of HLBW on glomerular sclerosis index (SI) of rats with DN. Values are mean $\pm \mathrm{SD}(n=5) .{ }^{\triangle} \mathrm{A} P<0.01$ versus the control group, ${ }^{\boldsymbol{\Lambda}} P<0.01$ versus the untreated diabetic group, and ${ }^{* *} P<0.01$ versus HLBW group.

proteins on the gel were transferred to nitrocellulose membranes. The membranes were then blocked with $5 \%$ fat-free dry milk in TBST or $0.5 \%$ bovine serum albumin (BSA) for $2 \mathrm{~h}$ at room temperature, followed by overnight incubation at $4^{\circ} \mathrm{C}$ with antibodies ( $47^{\mathrm{phox}}$, phosphorylated PKC$\alpha$, fibronectin, and $\beta$-actin) (Abcam, Hong kong, China).
After washed by TBST three times, the membranes were lucifugally incubated with the dylight 800-labeled antibody to rabbit $\operatorname{IgG}(\mathrm{H}+\mathrm{L})$ (KPL Company, Hongkong, China) at room temperature for $1 \mathrm{~h}$. Then, the membranes were lucifugally washed with TBST three times. Immunoreactive proteins were detected by near infrared double color laser imaging system (Odyssey, Lincoln, USA). Band densities were determined by Bio-Rad Quantity One software and quantified as the ratio between $\mathrm{OD}$ value of target band to OD value of $\beta$-actin.

2.10. Quantitative RT-PCR Analysis. Total RNA was extracted from the renocortical tissue with Trizol reagent according to the manufacturer's instructions. RNA purity and concentration were measured by a Nucleic Acid/Protein Analyzer (Thermo, Rockford, USA). The extracted total RNA (1 $\mu \mathrm{g})$ was reverse transcribed with PrimeScript RT reagent Kit (TaKaRa Company, Dalian, China) on a Mastercycler gradient PCR apparatus (Eppendorf Company, Hamburg, Germany). The cDNA was kept at $-20^{\circ} \mathrm{C}$ prior to PCR amplification. Real-time PCR reactions were performed in 48well optical PCR plates using SYBR Premix Ex Taq (TaKaRa Company, Dalian, China) on an Applied Biosystems StepOne Real-Time PCR System (Stepone, Foster City, USA). A $2^{-\Delta \Delta C T}$ was used for analyzing the data. Primer sequences are listed in Table 1. 


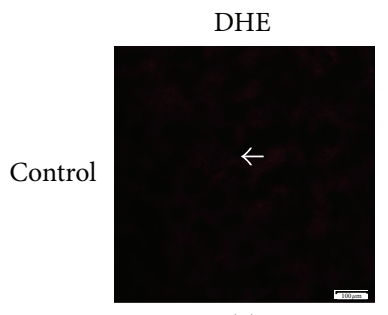

(a)

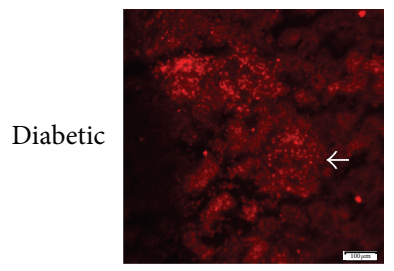

(b)

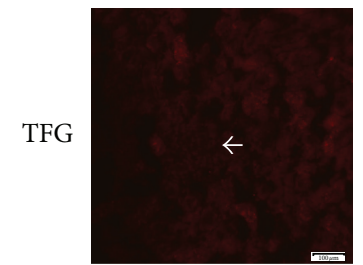

(c)

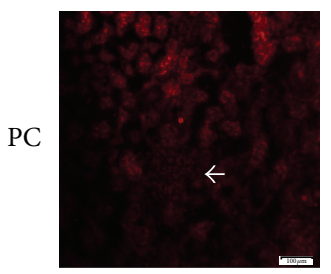

(d)

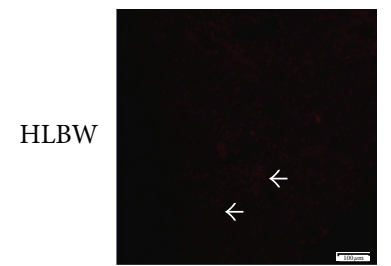

(e)

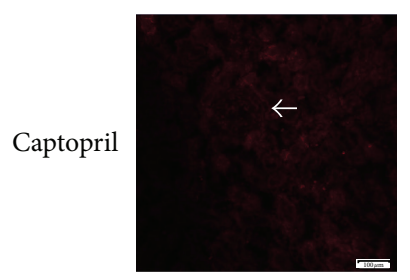

(f)

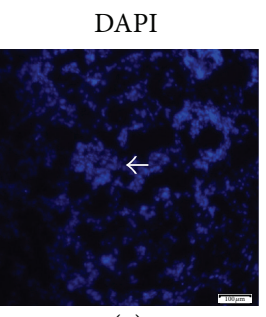

(g)

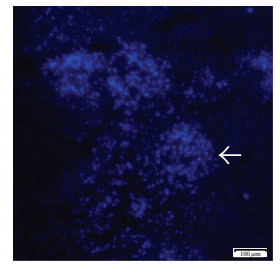

(h)

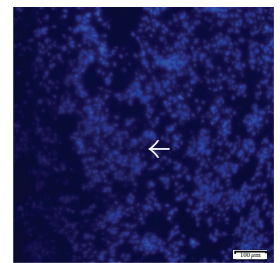

(i)

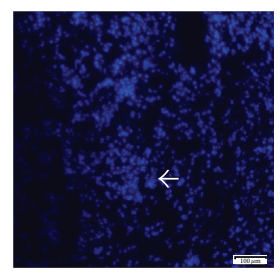

(j)

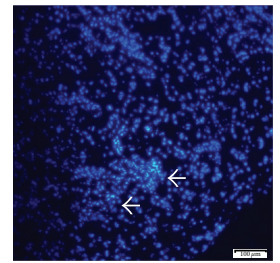

(k)

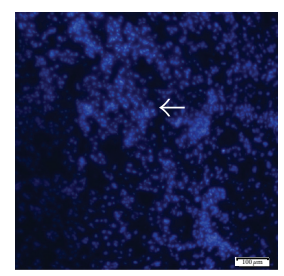

(l)

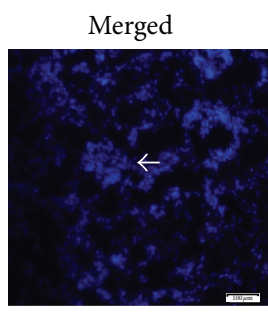

(m)

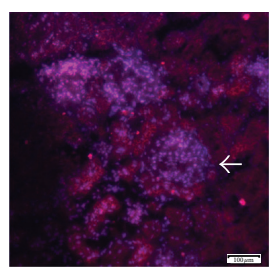

(n)

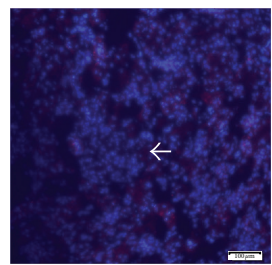

(o)

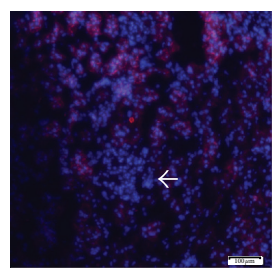

(p)

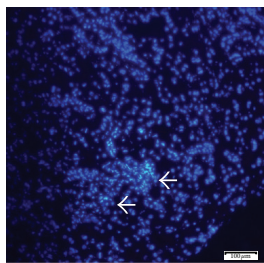

(q)

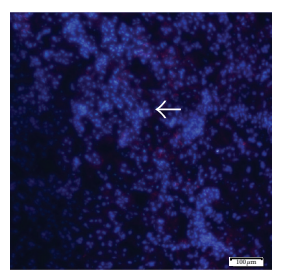

(r)

FIGURE 3: Dihydroethidium staining in the glomeruli from the rats in different groups. (original magnification: $\times 100)$. (a)-(f) Visualization of ROS in the glomeruli using DHE stains. (g)-(l) Visualization of nucleus in the glomeruli using DAPI stains. (m)-(r) The superimposed pictures of different groups. (Arrows are pointing at the glomerulus.)

2.11. Statistical Analysis. All data are presented as mean \pm standard deviation (SD) and analyzed by SPSS19.0 Statistical Software. Statistical significance was determined by one-way analysis of variance (ANOVA). Data with equal variances were not assumed followed by Dunnett's T3 test, while data with equal variances assumed followed by LSD test. A probability of less than 0.05 was considered to be statistically significant. 


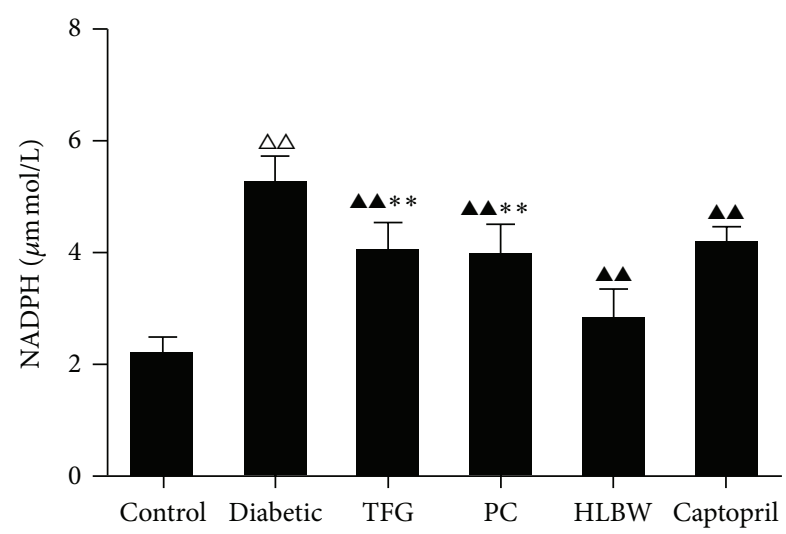

FIGURE 4: The effect of HLBW on renal NADPH activity of rats with $\mathrm{DN}$. Values are mean $\pm \mathrm{SD}(n=8) .{ }^{\triangle} P<0.01$ versus the control group, ${ }^{\mathbf{\Delta}} P<0.01$ versus the untreated diabetic group, and ${ }^{* *} P<$ 0.01 versus HLBW group.

\section{Results}

3.1. HLBW Improved the Glucose Tolerance of Rats with $D N$. Rats with untreated DN showed severe hyperglycemia characterized by elevated fasting and postprandial plasma glucose levels $(P<0.01)$. However, treatment with HLBW and its single components, as well as Captopril, significantly decreased plasma glucose levels compared with those of untreated diabetic rats $(P<0.01)$ (Table 2$)$.

3.2. HLBW Improved Plasma Lipid Profiles of Rats with DN. As shown in Table 3, rats with untreated DN showed severe dyslipidemia. The serum TC, TG, and LDL-C levels increased compared with those of control rats $(P<0.01)$. Treatment with HLBW and its single components markedly alleviated hyperlipidemia in rats with DN $(P<0.01, P<0.05$, resp.). However, Captopril did not show any beneficial effect on dyslipidemia when compared with diabetic rats.

3.3. HLBW Improved Renal Function and Proteinuria of Rats with DN. As shown in Table 4, rats with untreated DN exhibited an elevation in the term of ratio of kidney to body weight, as well as severe renal dysfunction and proteinuria. The BUN, SCr, urinary total protein, and albumin concentrations increased significantly in comparison to those of control rats $(P<0.01)$. However, treatment with HLBW and its single components, as well as Captopril, significantly reduced the ratio of kidney to body weight and reversed renal dysfunction and proteinuria $(P<0.01)$.

3.4. HLBW Improved Renal Morphology Changes of Rats with DN. As shown in Figure 1, renal tissues of rats with untreated DN showed remarkable glomerular hypertrophy and fibrosis, hyperplasia of mesangial area, and effacement of the podocyte foot processes. As shown in Figure 2, SI of rats with untreated DN elevated significantly. However, treatment with HLBW restored these morphology changes and SI level. Treatment with either TFG or PC also attenuated glomerular hypertrophy and fibrosis, mesangial hyperplasia, podocyte foot processes effacement, and glomerular SI level.

3.5. HLBW Decreased Renal Superoxide Anion Production of Rats with DN. As shown in Figure 3, a high level of DHE fluorescence, indicating the increased superoxide anion production $[23,24]$, was observed in renal tissues of $\mathrm{DN}$ rats. However, treatment with HLBW and its single components, as well as Captopril, significantly reduced the level of DHE fluorescence in renal tissues of rats with DN.

3.6. HLBW Decreased Renal NADPH Activity of Rats with DN. As shown in Figure 4, the activity of renal NADPH was much higher in DN rats than that in control rats $(P<0.01)$. After the treatment with HLBW, TFG, PC, or Captopril, the activity of renal NADPH was significantly decreased in rats with DN $(P<0.01)$. Furthermore, a significant difference in renal NADPH activity was identified between HLBW and its single components, indicating a better renal NADPH decreasing activity of HLBW than that of TFG and PC $(P<0.01)$.

3.7. HLBW Decreased Renal PKC- $\alpha$, Phosphorylated PKC- $\alpha$, p47 $7^{\text {phox }}$, and Fibronectin Gene Expressions of Rats with DN. As shown in Figures 5 and 6 , renal $\mathrm{p} 47^{\text {phox }}$ protein level and mRNA concentration significantly increased in DN rats compared with those in control rats $(P<0.01)$. However, there was a significant reduction in the expression of $\mathrm{p} 47^{\text {phox }}$ protein and mRNA in all the treatment groups $(P<0.01)$. Moreover, a marked reduction in the expression of $\mathrm{p} 47^{\text {phox }}$ protein and mRNA was identified after HLBW treatment in contrast to TFG or PC treatment alone $(P<0.01, P<0.05)$.

As shown in Figures 5 and 6, renal phosphorylated PKC$\alpha$ protein level increased significantly in rats with untreated DN $(P<0.01)$. HLBW, TFG, PC and Captopril treatment significantly reduced the expression of phosphorylated PKC$\alpha$ protein $(P<0.01)$. Additionally, HLBW, better than TFG or PC alone, significantly decreased phosphorylated PKC- $\alpha$ protein expression $(P<0.01)$. However, in terms of PKC$\alpha$ mRNA expression, no significant difference was identified between the groups.

With regard to Fibronectin, which is a marker of renal fibrosis, rats with untreated DN exhibited a significant increase of renal Fibronectin protein expression $(P<0.01)$. HLBW, treatment with TFG, PC, and Captopril significantly reduced the expression of renal fibronectin protein $(P<$ $0.01)$. HLBW also better than TFG or PC alone, significantly decreased renal expression of fibronectin protein $(P<0.01)$.

\section{Discussion}

HLBW is a Chinese herbal prescription that is just composed of two herbs, TFG and PC. Previous evidence from animal and clinical studies has suggested that TFG has the potential to treat hyperglycemia, hyperlipidemia, and renal disease [7-10, 18]. However, the comparisons between the effect of HLBW and its single components on DN have rarely been reported. 


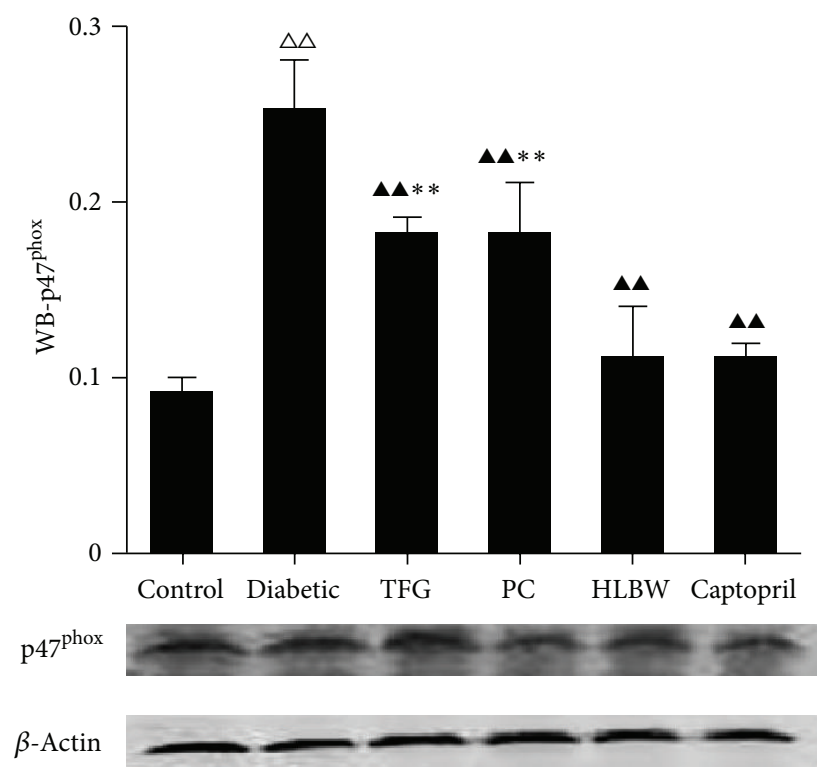

(a)

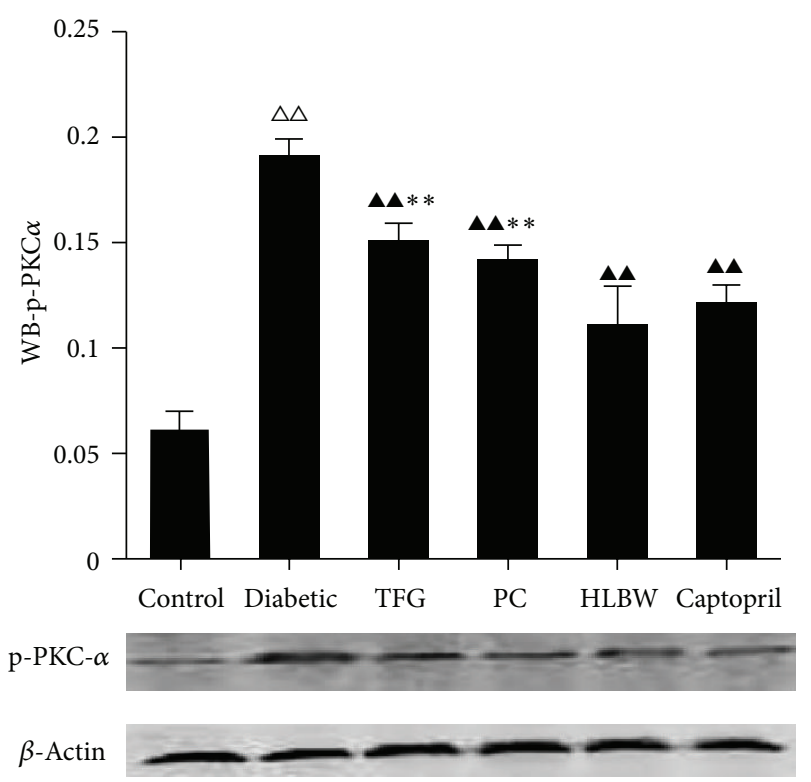

(b)

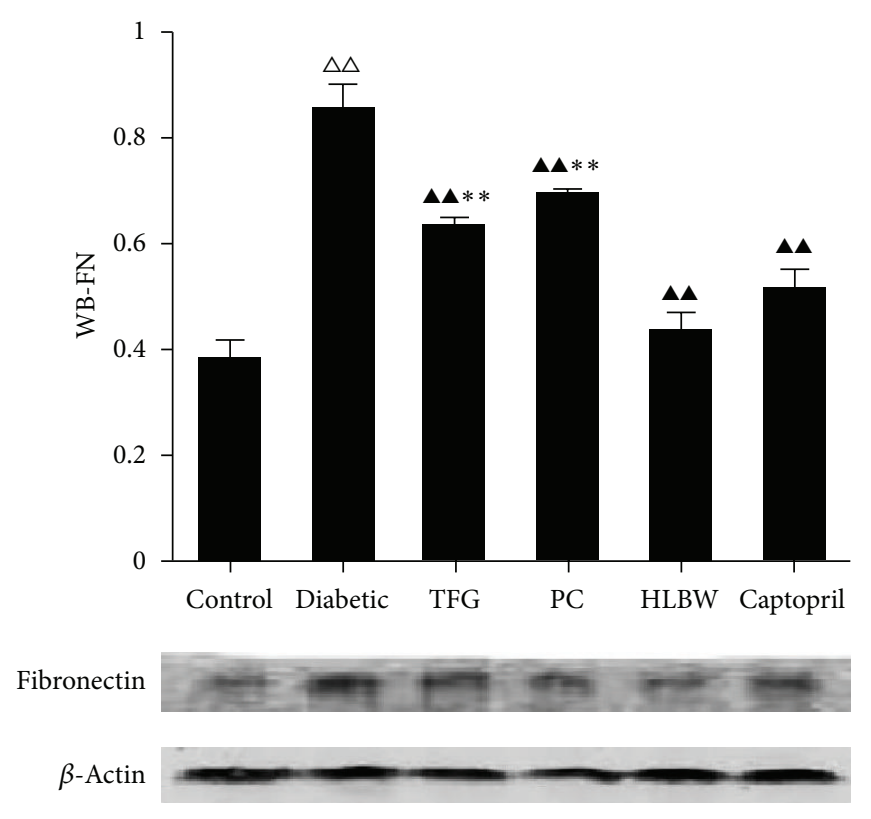

(c)

FIGURE 5: The effect of HLBW on the expression of renal proteins: (a) p47 ${ }^{\text {phox }}$; (b) p-PKC- $\alpha$; (c) fibronectin. Values are mean \pm SD $(n=8)$. ${ }^{\triangle} \triangle P<0.01$ versus the control group, ${ }^{\Delta \mathbf{\Delta}} P<0.01$ versus the untreated diabetic group, and ${ }^{* *} P<0.01$ versus HLBW group.

In our study, the rat model of type 2 diabetes was successfully established by a high-fat diet accompanied by intravenous injection of relatively small doses of STZ. The animals manifested the characteristics of hyperglycemia and hyperlipidemia. BUN, SCr, 24-hour urinary total protein, and albumin concentrations significantly increased at the same time, which indicated the development of DN. HLBW showed hypoglycemia, hypolipidemia, and renal protection against DN. Meanwhile, such the beneficial outcomes were also confirmed in DN rats treated with TFG or PC alone. With regard to renal morphology changes, the untreated diabetic rats were characteristized of remarkable glomerular expansion and fibrosis, extracellular matrix (ECM) accumulation, and effacement of the foot processes. HLBW, also similar to TFG and PC, attenuated these histopathological abnormalities in renal tissue in diabetic rats. However, HLBW, better than TFG and PC, reduced the expression of renal fibronectin protein and the level of SI. Since fibronectin is a predominant matrix protein representing the degree of renal fibrosis and SI represents the extent of glomerular sclerosis, this result indicates that HLBW may be promising at ameliorating renal fibrosis. 


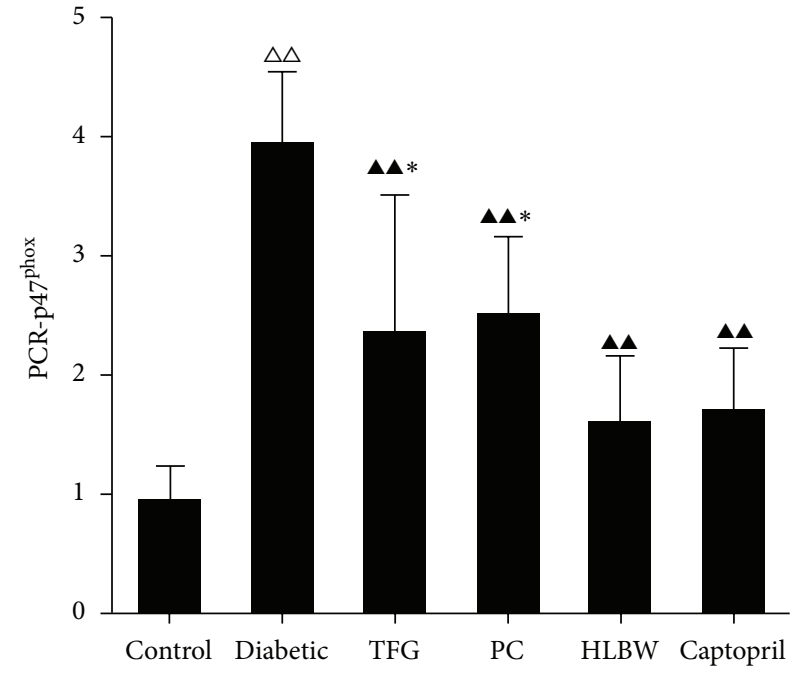

(a)

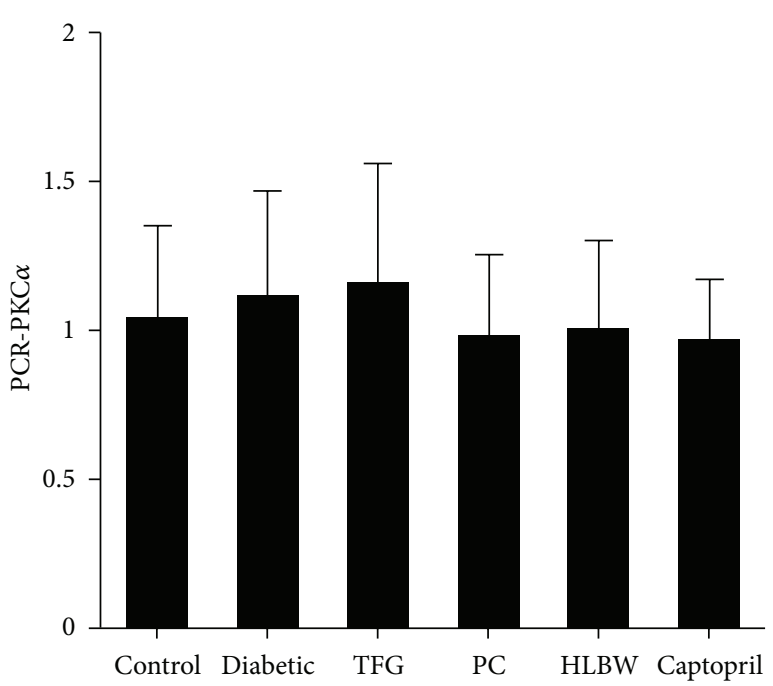

(b)

FIgURE 6: The effect of HLBW on the expression of renal p47 $7^{\text {phox }}$ (a) and PKC- $\alpha$ (b) mRNA. Values are mean \pm SD ( $\left.n=8\right) .{ }^{\Delta \triangle} P<0.01$ versus the control group, ${ }^{\mathbf{\Lambda}} P<0.01$ versus the untreated diabetic group, and ${ }^{*} P<0.05$ versus HLBW group.

In diabetic patients, hyperglycemia and hyperlipidemia enhance the oxidative stress which is involved in the mechanism of diabetic vasculopathy. The oxidative stress can also aggravate the glucolipid metabolism disorder, thereby forming a vicious circle $[5,6,25]$. Excessive reactive oxygen species (ROS) generated by oxidative stress not only induces oxidative damage via peroxidation of the biomacromolecule but also interferes with cell signal transduction via serving as a second messenger [26]. To evaluate superoxide production in the kidneys, dihydroethidium (DHE) staining is often performed. In the presence of superoxide, DHE is changed into ethidium bromide, which binds to DNA and exhibits red fluorescence in the nucleus [27-29]. In our study, a massive increase in superoxide anion generation was identified in the diabetic renal tissues. It was attenuated by HLBW and its single components. However, HLBW treatment, compared with TFG or PC, showed the maximum decreasing of superoxide production, indicating the efficient oxidative stress protection in DN.

In order to elucidate the protection mechanisms of HLBW against oxidative stress in renal tissues, we further evaluated the gene expressions involved in the production of ROS. Among the multiple sources in the diabetic kidney, ROS derived from NADPH oxidase is crucial to the development of $\mathrm{DN}[30,31]$. Accordingly, we further examined the activity of NADPH oxidase and the gene expressions of its upstream regulator, protein kinase $\mathrm{C}-\alpha$ (PKC- $\alpha$ ), and $\mathrm{p} 47^{\text {phox }}$, which are the regulatory subunit of NADPH oxidase [26]. The results showed that HLBW significantly inhibited NADPH oxidase activity and $\mathrm{p} 47^{\text {phox }}$ gene expression in renal tissues of diabetic rats. HLBW also decreased phosphorylated PKC$\alpha$ protein expression. These effects were also superior to that observed in DN rats treated with TFG or PC alone. However, no significant difference was identified between the groups regarding the expression of $\mathrm{PKC}-\alpha \mathrm{mRNA}$. It might give a hint that the effect of HLBW on PKC- $\alpha$ is posttranscription.
Therefore, our study firstly demonstrated the inhibitory effect of HLBW on PKC- $\alpha / \mathrm{NADPH}$ oxidase signaling pathway, which may attribute to the reduction of ROS production in renal tissues.

In summary, our study demonstrates that HLBW and its single components, TFG and PC, improve renal function and ameliorate renal histopathological alterations in type 2 diabetic rats. The mechanism may be related to reducing oxidative stress via PKC- $\alpha / \mathrm{NADPH}$ oxidase signaling pathway. Moreover, HLBW exhibits a better efficacy than TFG or PC alone on protecting against oxidative stress in DN, which indicates a theory of prescription compatibility in traditional Chinese medicine.

\section{Conflict of Interests}

The authors declare that they have no conflict of interests to disclose.

\section{Authors' Contribution}

Lishan Zhou and Hui Dong contributed equally to this work.

\section{Acknowledgment}

This work was supported by the National Natural Science Foundation of China (no. 81273683).

\section{References}

[1] W. Y. Yang, J. M. Lu, J. P. Weng et al., "Prevalence of diabetes among men and women in China," The New England Journal of Medicine, vol. 362, no. 12, pp. 1090-1101, 2010.

[2] American Diabetes Association, "Economic consequences of diabetes mellitus in the U.S. in 1997," Diabetes Care, vol. 21, no. 2, pp. 296-309, 1998. 
[3] C. A. Jones, A. S. Krolewski, J. Rogus, J. L. Xue, A. Collins, and J. H. Warram, "Epidemic of end-stage renal disease in people with diabetes in the United States population: do we know the cause?" Kidney International, vol. 67, no. 5, pp. 1684-1691, 2005.

[4] K. Winiarska, K. Szymanski, P. Gorniak, M. Dudziak, and J. Bryla, "Hypoglycaemic, antioxidative and nephroprotective effects of taurine in alloxan diabetic rabbits," Biochimie, vol. 91, no. 2, pp. 261-270, 2009.

[5] P. Manna, M. Sinha, and P. C. Sil, "Prophylactic role of arjunolic acid in response to streptozotocin mediated diabetic renal injury: activation of polyol pathway and oxidative stress responsive signaling cascades," Chemico-Biological Interactions, vol. 181, no. 3, pp. 297-308, 2009.

[6] P. Manna, M. Sinha, and P. C. Sil, "Taurine plays a beneficial role against cadmium-induced oxidative renal dysfunction," Amino Acids, vol. 36, no. 3, pp. 417-428, 2009.

[7] F. R. Lu, L. Shen, Y. Qin, L. Gao, H. Li, and Y. Dai, "Clinical observation on Trigonella foenum-graecum L. total saponins in combination with sulfonylureas in the treatment of type 2 diabetes mellitus," Chinese Journal of Integrative Medicine, vol. 14, no. 1, pp. 56-60, 2008.

[8] A. Srichamroen, C. J. Field, A. B. R. Thomson, and T. K. Basu, "The modifying effects of galactomannan from Canadiangrown fenugreek (Trigonella foenum-graecum L.) on the glycemic and lipidemic status in rats," Journal of Clinical Biochemistry and Nutrition, vol. 43, no. 3, pp. 167-174, 2008.

[9] C. Broca, V. Breil, C. Cruciani-Guglielmacci et al., "Insulinotropic agent ID-1101 (4-hydroxyisoleucine) activates insulin signaling in rat," American Journal of Physiology, vol. 287, no. 3, pp. E463-E471, 2004.

[10] L. Jetté, L. Harvey, K. Eugeni, and N. Levens, "4-Hydroxyisoleucine: a plant-derived treatment for metabolic syndrome," Current Opinion in Investigational Drugs, vol. 10, no. 4, pp. 353358, 2009.

[11] K. Bapat, G. J. Chintalwar, U. Pandey et al., "Preparation and in vitro evaluation of radioiodinated bakuchiol as an anti tumor agent," Applied Radiation and Isotopes, vol. 62, no. 3, pp. 389393, 2005.

[12] N. A. Khatune, M. E. Islam, M. E. Haque, P. Khondkar, and M. M. Rahman, "Antibacterial compounds from the seeds of Psoralea corylifolia," Fitoterapia, vol. 75, no. 2, pp. 228-230, 2004.

[13] Z. Xiong, D. Wang, Y. Xu, and F. Li, “Osteoblastic differentiation bioassay and its application to investigating the activity of fractions and compounds from Psoralea corylifolia L," Pharmazie, vol. 58, no. 12, pp. 925-928, 2003.

[14] U. N. Tripathi and D. Chandra, "The plant extracts of Momordica charantia and Trigonella foenum graecum have antioxidant and anti-hyperglycemic properties for cardiac tissue during diabetes mellitus," Oxidative Medicine and Cellular Longevity, vol. 2, no. 5, pp. 290-296, 2009.

[15] R. Sangeetha, "Activity of superoxide dismutase and catalase in fenugreek (Trigonella foenum-graecum) in response to carbendazim," Indian Journal of Pharmaceutical Sciences, vol. 72, no. 1, pp. 116-118, 2010.

[16] Y. T. Soon, J. Gruber, P. W. Kim, and B. Halliwell, "Psoralea corylifolia L. inhibits mitochondrial complex I and proteasome activities in SH-SY5Y cells," Annals of the New York Academy of Sciences, vol. 1100, pp. 486-496, 2007.

[17] S. Jan, T. Parween, T. O. Siddiqi et al., "Anti-oxidant modulation in response to gamma radiation induced oxidative stress in developing seedlings of Psoralea corylifolia L," Journal of Environmental Radioactivity, vol. 113, pp. 142-149, 2012.

[18] S. Thakran, M. R. Siddiqui, and N. Z. Baquer, "Trigonella foenum graecum seed powder protects against histopathological abnormalities in tissues of diabetic rats," Molecular and Cellular Biochemistry, vol. 266, no. 1-2, pp. 151-159, 2004.

[19] Y. C. Kim, H. Oh, B. S. Kim et al., "In vitro protein tyrosine phosphatase $1 \mathrm{~B}$ inhibitory phenols from the seeds of Psoralea corylifolia," Planta Medica, vol. 71, no. 1, pp. 87-89, 2005.

[20] L. J. Ma and A. B. Fogo, "Model of robust induction of glomerulosclerosis in mice: importance of genetic background," Kidney International, vol. 64, no. 1, pp. 350-355, 2003.

[21] H. Parfenova, C. W. Leffler, S. Basuroy, J. Liu, and A. L. Fedinec, "Antioxidant roles of heme oxygenase, carbon monoxide, and bilirubin in cerebral circulation during seizures," Journal of Cerebral Blood Flow and Metabolism, vol. 32, pp. 1024-1034, 2012.

[22] H. Hendarto, T. Inoguchi, Y. Maeda et al., "GLP-1 analog liraglutide protects against oxidative stress and albuminuria in streptozotocin-induced diabetic rats via protein kinase A-mediated inhibition of renal NAD(P)H oxidases," Metabolism, vol. 61, no. 10, pp. 1422-1434, 2012.

[23] O. Kitamura, K. Uemura, H. Kitamura, H. Sugimoto, A. Akaike, and T. Ono, "Serofendic acid protects from iodinated contrast medium and high glucose probably against superoxide production in LLC-PK1 cells," Clinical and Experimental Nephrology, vol. 13, no. 1, pp. 15-24, 2009.

[24] J. A. Cabrera, E. A. Ziemba, R. Colbert et al., "Uncoupling protein-2 expression and effects on mitochondrial membrane potential and oxidant stress in heart tissue," Translational Research, vol. 159, no. 5, pp. 383-390, 2012.

[25] K. Winiarska, K. Szymanski, P. Gorniak, M. Dudziak, and J. Bryla, "Hypoglycaemic, antioxidative and nephroprotective effects of taurine in alloxan diabetic rabbits," Biochimie, vol. 91, no. 2, pp. 261-270, 2009.

[26] R. P. Brandes and J. Kreuzer, "Vascular NADPH oxidases: molecular mechanisms of activation," Cardiovascular Research, vol. 65, no. 1, pp. 16-27, 2005.

[27] S. Sato, H. Kawamura, M. Takemoto et al., "Halofuginone prevents extracellular matrix deposition in diabetic nephropathy," Biochemical and Biophysical Research Communications, vol. 379, no. 2, pp. 411-416, 2009.

[28] C. C. Lin, L. T. Lin, M. H. Yen et al., "Renal protective effect of Xiao-Chai-Hu-Tang on diabetic nephropathy of type 1diabetic mice," Evidence-Based Complementary and Alternative Medicine, vol. 2012, Article ID 984024, 11 pages, 2012.

[29] L. Pernomian, M. Santos Gomes, C. Baraldi Araujo Restini et al., "The role of reactive oxygen species in the modulation of the contraction induced by angiotensin II in carotid artery from diabetic rat," European Journal of Pharmacology, vol. 678, no. 13, pp. 15-25, 2012.

[30] J. M. Forbes, M. T. Coughlan, and M. E. Cooper, "Oxidative stress as a major culprit in kidney disease in diabetes," Diabetes, vol. 57, no. 6, pp. 1446-1454, 2008.

[31] H. Toba, N. Sawai, M. Morishita et al., "Chronic treatment with recombinant human erythropoietin exerts renoprotective effects beyond hematopoiesis in streptozotocin-induced diabetic rat," European Journal of Pharmacology, vol. 612, no. 1-3, pp. 106-114, 2009. 


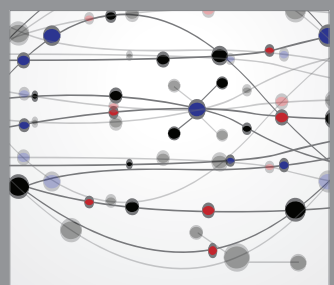

The Scientific World Journal
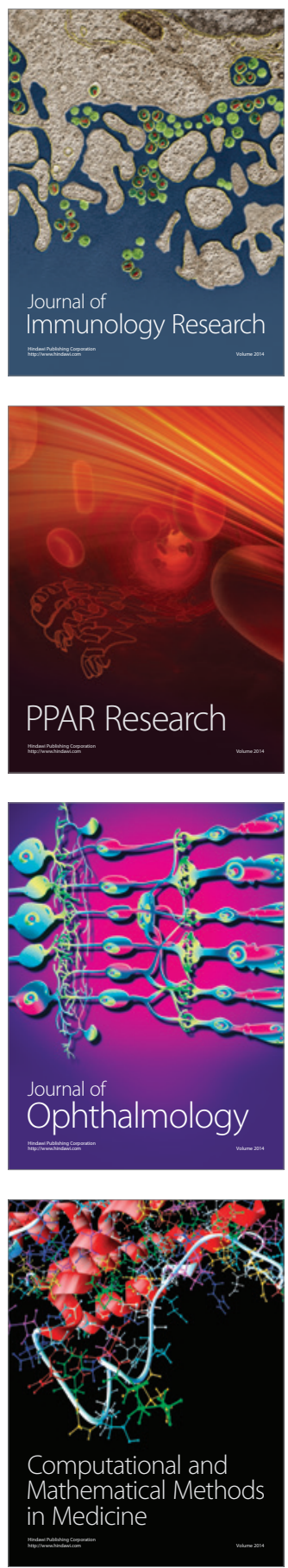

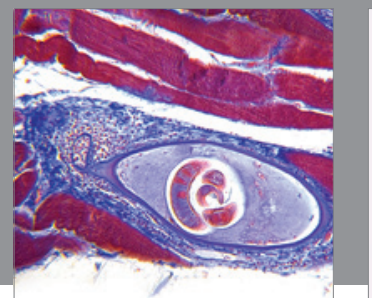

Gastroenterology

Research and Practice
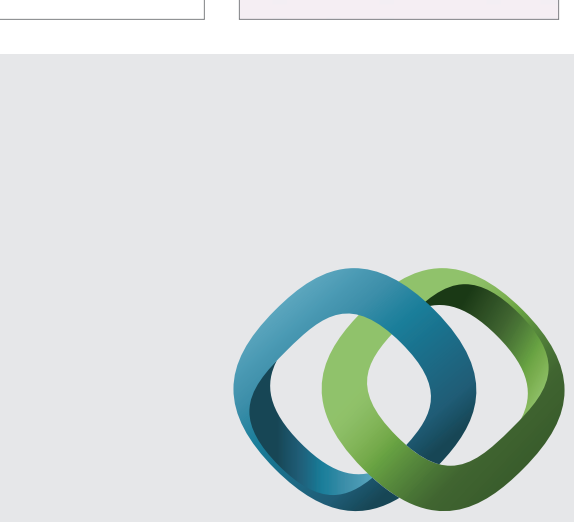

\section{Hindawi}

Submit your manuscripts at

http://www.hindawi.com
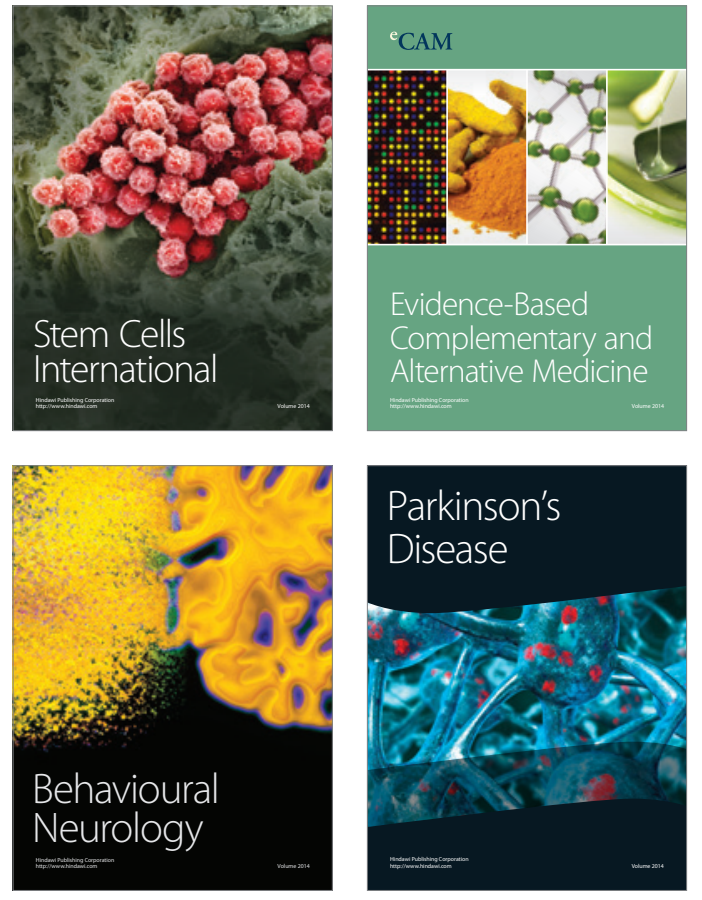
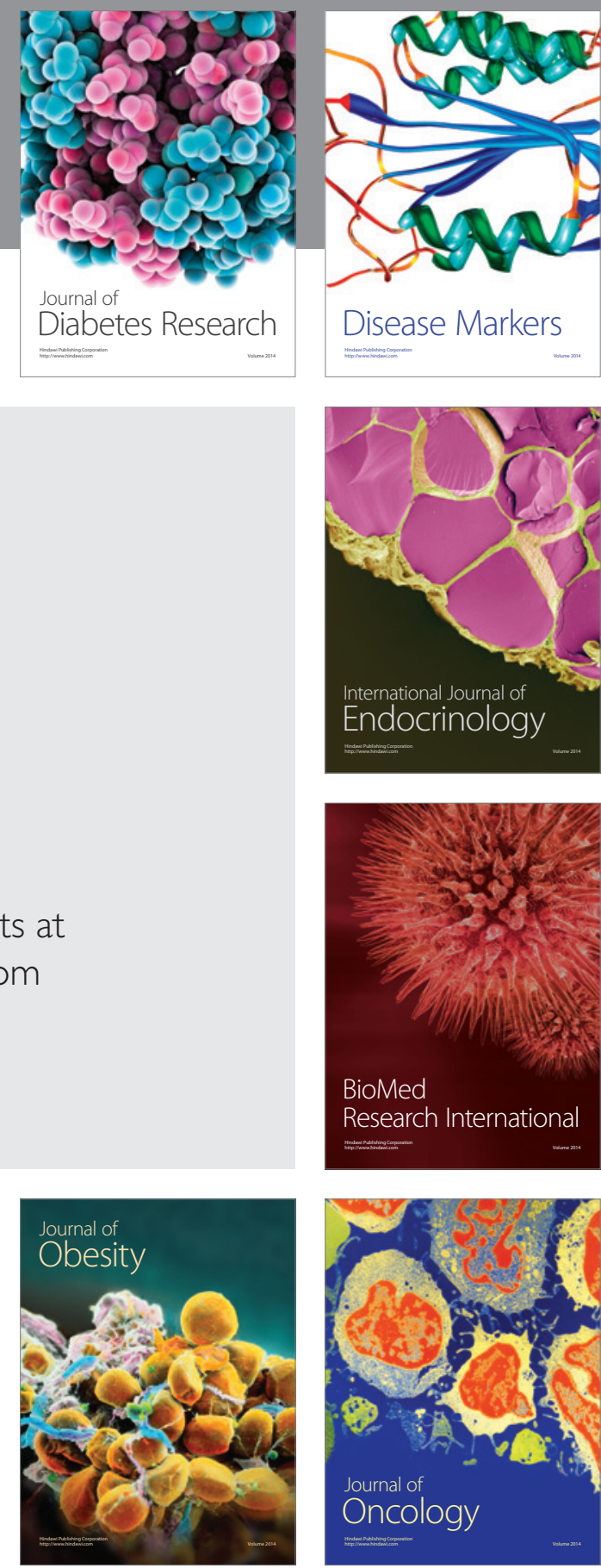

Disease Markers
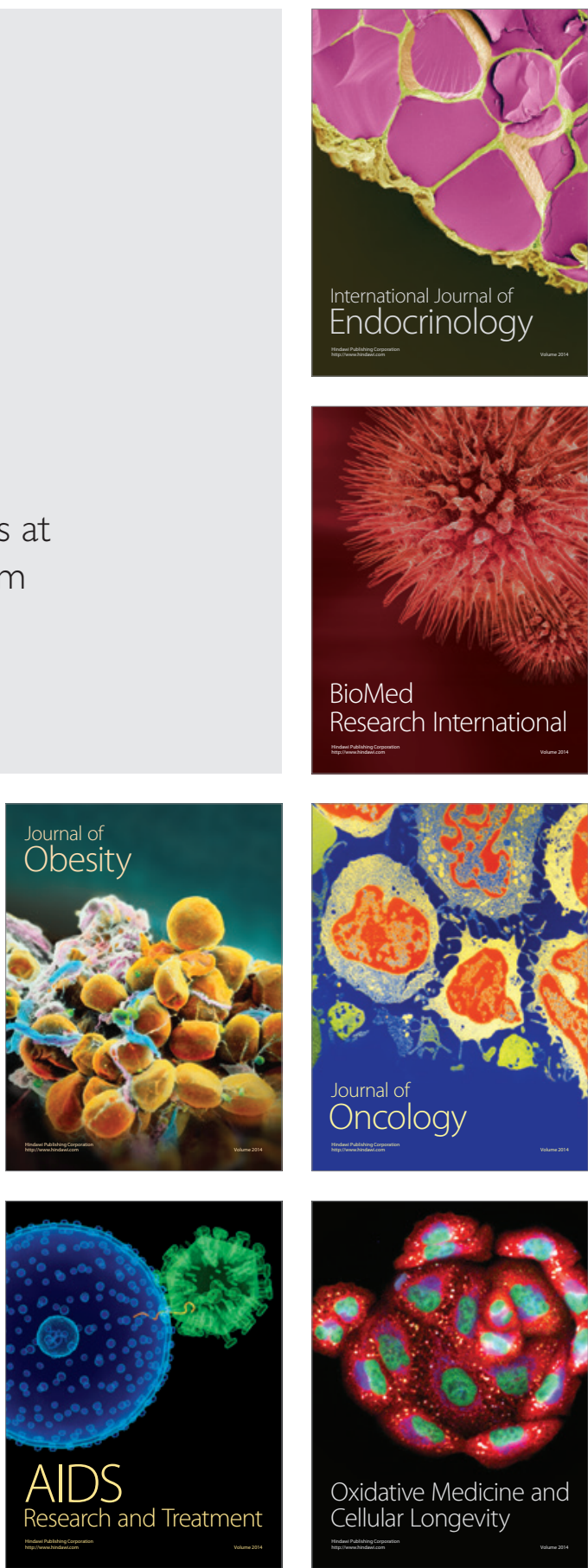